\title{
A Polynomial Representation and a Unique Code of a Simple Undirected Graph
}

\author{
Shamik Ghosh, ${ }^{1}$ Raibatak Sen Gupta, ${ }^{1}$ and M. K. Sen ${ }^{2}$ \\ ${ }^{1}$ Department of Mathematics, Jadavpur University, Kolkata 700032, India \\ ${ }^{2}$ Department of Pure Mathematics, University of Calcutta, Kolkata 700019, India \\ Correspondence should be addressed to Shamik Ghosh; sghosh@math.jdvu.ac.in
}

Received 30 June 2013; Accepted 29 July 2013

Academic Editors: E. Bannai, L. Clark, and D. S. Kim

Copyright (c) 2013 Shamik Ghosh et al. This is an open access article distributed under the Creative Commons Attribution License, which permits unrestricted use, distribution, and reproduction in any medium, provided the original work is properly cited.

We introduce a representation of simple undirected graphs in terms of polynomials and obtain a unique code for a simple undirected graph.

\section{Introduction}

Let $M$ be the set of all positive integers greater than 1. Let $n \in$ $M$ and $V(n)$ be the set of all divisors of $n$, greater than 1 . Define a simple undirected graph $G(n)=(V, E)$ with the vertex set $V=V(n)$ and any two distinct vertices $a, b \in V$ are adjacent if and only if $\operatorname{gcd}(a, b)>1$. From an observation in [1], it follows that any simple undirected graph is isomorphic to an induced subgraph of $G(n)$ for some $n \in M$. For the sake of completeness and further use of the construction we provide a sketch of the proof below. Throughout the paper by a graph, we mean a simple undirected graph.

Theorem 1. Let $G$ be a graph. Then $G$ is isomorphic to an induced subgraph of $G(n)$ for some $n \in M$.

Proof. Let $G=(V, E)$ be a graph. Let $\left\{C_{1}, C_{2}, \ldots, C_{k}\right\}$ be the set of all maximal cliques of $G$. For $i=1,2, \ldots, k$, let $p_{i}$ be the $i$ th prime. For each $v \in V$, define $s_{1}(v)=\prod\left\{p_{j} \mid v \in C_{j}\right\}$. Now in order to make the values of $s_{1}(v)$ distinct for distinct vertices, we modify $s_{1}(v)$ by using different powers of primes $p_{j}$, if required. For each $v \in V$, let $s(v)$ be the modified value of $s_{1}(v)$. Let $n$ be the least common multiple of $\{s(v) \mid v \in V\}$. Now it is clear that for any $u, v \in V$,

$u$ is adjacent to $v$ in $G \Longleftrightarrow u, v \in C_{i}$

for some $i \in\{1,2, \ldots, k\}$
$\Longleftrightarrow p_{i}$ is a factor of both $s(u)$ and $s(v)$

for some $i \in\{1,2, \ldots, k\}$

$\Longleftrightarrow \operatorname{gcd}(s(u), s(v))>1 \Longleftrightarrow s(u)$ is adjacent to

$s(v)$ in $G(n)$.

Thus, $G$ is isomorphic to the subgraph of $G(n)$ induced by the set $\{s(v) \mid v \in V\}$ of vertices of $G(n)$.

Example 2. Consider the graph $G=(V, E)$ in Figure 1. The maximal cliques of $G$ are $C_{1}=\left\{v_{1}, v_{2}, v_{3}, v_{4}\right\}, C_{2}=$ $\left\{v_{2}, v_{3}, v_{4}, v_{5}, v_{6}\right\}, C_{3}=\left\{v_{7}, v_{8}, v_{10}\right\}, C_{4}=\left\{v_{9}, v_{10}\right\}$, and $C_{5}=$ $\left\{v_{10}, v_{11}\right\}$. We assign the $i$ th prime to the clique $C_{i}$ for $i=$ $1,2, \ldots, 5$. Then for each $v \in V$, we compute $s_{1}(v)$ and $s(v)$ as in the proof of Theorem 1 (Table 1).

So $G$ is isomorphic to the subgraph of $G(n)$ induced by the set $\{2,3,5,6,7,9,11,12,18,25,385\}$ of vertices of $G(n)$, where $n=2^{2} \cdot 3^{2} \cdot 5^{2} \cdot 7 \cdot 11=69300$.

\section{Main Results}

It is important to note that instead of taking all maximal cliques of $G$ in Theorem 1, it is sufficient to consider a set of cliques of $G$ which covers both vertices and edges of $G$. 
TABLE 1

\begin{tabular}{lccccccccccc}
\hline$v$ & $v_{1}$ & $v_{2}$ & $v_{3}$ & $v_{4}$ & $v_{5}$ & $v_{6}$ & $v_{7}$ & $v_{8}$ & $v_{9}$ & $v_{10}$ & $v_{11}$ \\
\hline$s_{1}(v)$ & 2 & $2 \cdot 3$ & $2 \cdot 3$ & $2 \cdot 3$ & 3 & 3 & 5 & 5 & 7 & $5 \cdot 7 \cdot 11$ \\
$s_{1}(v)$ & 2 & 6 & 6 & 6 & 3 & 3 & 5 & 5 & 7 & 385 \\
$s(v)$ & 2 & $2 \cdot 3$ & $2^{2} \cdot 3$ & $2 \cdot 3^{2}$ & 3 & $3^{2}$ & 5 & $5^{2}$ & 7 & 11 \\
$s(v)$ & 2 & 6 & 12 & 18 & 3 & 9 & 5 & 25 & 7 & 385 & 11 \\
\hline
\end{tabular}

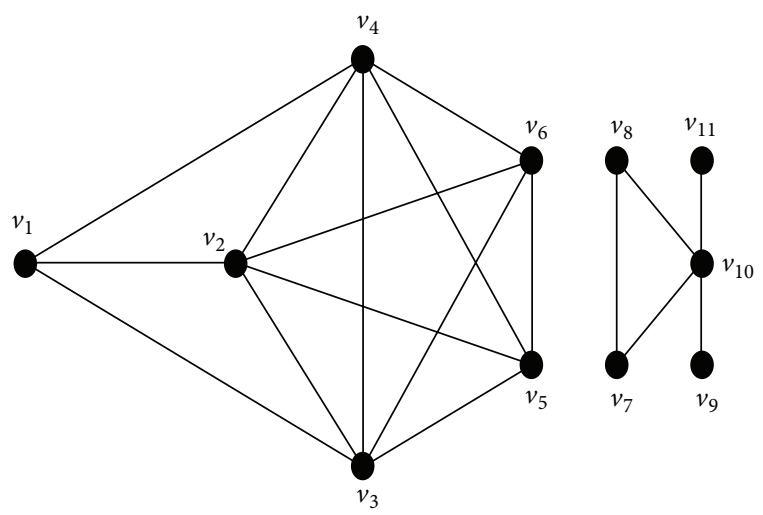

Figure 1: The graph $G$ in Example 2.

Definition 3. Let $G=(V, E)$ be a graph. A set $S$ of cliques of $G$ is called a total clique covering of $G$ if $S$ covers both $V$ and $E$. The minimum size of a total clique covering of $G$ is called the total clique covering number of $G$. We denote it by $\theta_{t}(G)$.

On the other hand, given a finite sequence of positive integers, one can construct a simple undirected graph as follows.

Definition 4. Let $\sigma=\left(a_{1}, a_{2}, \ldots, a_{m}\right)$ be a finite sequence of positive integers. Then corresponding to this sequence, define a graph $G[\sigma]=(V, E)$, where $V=\left\{v_{1}, v_{2}, \ldots, v_{m}\right\}, v_{i}$ corresponds to $a_{i}\left(a_{i}\right.$ is called the label of $\left.v_{i}\right)$ for $i=1,2, \ldots, m$, and $v_{i} v_{j} \in E$ if and only if $i \neq j$ and $\operatorname{gcd}\left(a_{i}, a_{j}\right)>1$. The graph $G[\sigma]$ is said to be realized by the sequence $\sigma$.

Now by Theorem 1 , every graph can be realized by some sequence of positive integers. Also in Definition 4, it is sufficient to take the entries $a_{i}$ of $\sigma$ square-free if $a_{i}>1$. Thus, for convenience, we specify the sequence in the following manner.

Definition 5. Let $G=(V, E)$ be a graph. Let $|V|>1$, and let the graph $G_{1}$ be obtained from $G$ by deleting isolated vertices of $G$, if there are any. Let $\sigma_{1}$ be a finite nondecreasing sequence of square-free positive integers greater than 1 such that $G_{1} \cong$ $G\left[\sigma_{1}\right]$. If $G$ has $s$ isolated vertices, then we prefix $s$ number of l's in $\sigma_{1}$ to obtain the sequence $\sigma$. For $s=0, \sigma=\sigma_{1}$. If $G$ is a graph with a single vertex, then $\sigma=(1)$. Then $G \cong G[\sigma]$ and the sequence $\sigma$ is called a coding sequence of $G$. The entries of $\sigma$, which are greater than 1 , are called nontrivial. Let $\lambda(\sigma)$ be the least common multiple of all nontrivial entries of $\sigma$.

Lemma 6. Let $\sigma=\left(\lambda_{1}, \lambda_{2}, \ldots, \lambda_{m}\right)$ be a coding sequence of a graph $G=(V, E)$. Let $V=\left\{v_{1}, v_{2}, \ldots, v_{m}\right\}$, where $v_{i}$ corresponds to $\lambda_{i}$ for all $i=1,2, \ldots, m$ (one writes $\lambda_{i}=\mu\left(v_{i}\right)$ ). Let $\left\{p_{1}, p_{2}, \ldots, p_{k}\right\}$ be the set of all distinct prime factors of $\lambda(\sigma)$.
Suppose $G$ has $s \geqslant 0$ isolated vertices. Define $S_{j}=\left\{v_{j}\right\}$ for $j=1,2, \ldots, s$ and for each $j=1,2, \ldots, k$, define $S_{s+j}=\left\{v_{i} \in\right.$ $V \mid p_{j}$ divides $\left.\mu\left(v_{i}\right)=\lambda_{i}\right\}$. Then $S=\left\{S_{j} \mid j=1,2, \ldots, s+k\right\}$ is a total clique covering of $G$ such that $v \in S_{i_{1}} \cap S_{i_{2}} \cap \cdots \cap S_{i_{r}}\left(i_{t}>s\right.$, $t=1,2, \ldots, r)$ and $v \notin S_{j}$ for all $j \in\{s+1, s+2, \ldots, s+k\} \backslash$ $\left\{i_{1}, i_{2}, \ldots, i_{r}\right\}$ if and only if $\mu(v)=p_{i_{1}} p_{i_{2}} \cdots p_{i_{r}}$ and for each $j=1,2, \ldots, s, v \in S_{j}$ if and only if $\mu(v)=1$.

Proof. We first note that $S_{j}$ is a clique containing only the vertex $v_{j}$ for $j=1,2, \ldots, s$. Now for each $j \in\{1,2, \ldots, k\}$, $p_{j}$ divides $\lambda_{i}$ for some $i \in\{s+1, s+2, \ldots, m\}$ as $p_{j}$ divides $\lambda(\sigma)$. Thus, $v_{i} \in S_{s+j}$. So $S_{j} \neq \emptyset$, for all $j=1,2, \ldots s+k$. Also for any two vertices $v_{r}, v_{t} \in S_{s+j}, p_{j}$ divides both $\lambda_{r}$ and $\lambda_{t}$. So $v_{r}$ and $v_{t}$ are adjacent in $G$. Thus, each $S_{j}$ is a clique of $G$. Again since $\left\{p_{1}, p_{2}, \ldots, p_{k}\right\}$ is the set of all prime factors of $\lambda(\sigma)$, each vertex $v \in V$ belongs to $S_{j}$ for some $j \in\{1,2, \ldots, s+k\}$. So $S$ covers $V$. Moreover, two vertices $v_{i}$ and $v_{j}$ are adjacent if and only if $\operatorname{gcd}\left(\lambda_{i}, \lambda_{j}\right)>1$. So $\lambda_{i}$ and $\lambda_{j}$ must have at least one common prime factor, say, $p_{r}$ and hence $v_{i}$ and $v_{j}$ both lie in $S_{s+r}$. So $S$ also covers $E$ and hence $S$ is a total clique covering of $G$. Now $v \in S_{i_{1}} \cap S_{i_{2}} \cap \cdots \cap S_{i_{r}}\left(i_{t}>s, t=1,2, \ldots, r\right)$ and $v \notin S_{j}$ for all $j \in\{s+1, s+2, \ldots, s+k\} \backslash\left\{i_{1}, i_{2}, \ldots, i_{r}\right\}$ if and only if $p_{i_{1}} p_{i_{2}} \cdots p_{i_{r}}$ divides $\mu(v)$ but $p_{j}$ does not divide $\mu(v)$ for all $j \in\{s+1, s+2, \ldots, s+k\} \backslash\left\{i_{1}, i_{2}, \ldots, i_{r}\right\}$. Now since nontrivial entries of $\sigma$ are square-free integers, $\mu(v)$ is a product of distinct primes and hence $\mu(v)=p_{i_{1}} p_{i_{2}} \cdots p_{i_{r}}$. By Definition 5, for each $j=1,2, \ldots, s, v \in S_{j}$ if and only if $\mu(v)=1$.

Definition 7. Let $\sigma=\left(\lambda_{1}, \lambda_{2}, \ldots, \lambda_{m}\right)$ be a coding sequence of a graph $G$. Then the total clique covering $S$ of $G$ as defined in Lemma 6 is called the total clique covering of $G$ corresponding to the sequence $\sigma$ and is denoted by $S_{\sigma}$.

Lemma 8. Let $G=(V, E)$ be a graph, and let $S$ be a total clique covering of $G$. Then there exists a coding sequence $\sigma$ of $G$ such that $S=S_{\sigma}$.

Proof. Let $S=\left\{C_{1}, C_{2}, \ldots, C_{s+k}\right\}$, where $\left|C_{i}\right|=1$ for $i=$ $1,2, \ldots, s, s \geqslant 0$. Let $p_{i}$ denote the $i$ th prime. We define a map $f: V \rightarrow \mathbb{N}$ by $f(v)=1$ if $v \in C_{1} \cup C_{2} \cup \cdots \cup C_{s}$ and $f(v)=p_{i_{1}} p_{i_{2}} \cdots p_{i_{r}}$ if $v \in C_{s+i_{1}} \cap C_{s+i_{2}} \cap \cdots \cap C_{s+i_{r}}$ (where $1 \leq r \leq k)$ and $v \notin C_{j}$ for all $j \in\{s+1, s+2, \ldots, s+k\} \backslash\left\{s+i_{1}, s+\right.$ $\left.i_{2}, \ldots, s+i_{r}\right\}$. Now $S$ being a total clique covering, every vertex is assigned a label in this way. Also note that two vertices $u, v$ are adjacent in $G$ if and only if they lie in some $C_{s+j} \in S$ and consequently, if and only if $f(u)$ and $f(v)$ have a common prime factor $p_{j}$. Let us arrange the vertices of $G$ according to the nondecreasing order of $\{f(v) \mid v \in V\}$ and define the sequence $\sigma$ by $\sigma=\left(f\left(v_{1}\right), f\left(v_{2}\right), \ldots, f\left(v_{m}\right)\right)$, where $f\left(v_{1}\right) \leq$ $f\left(v_{2}\right) \leq \cdots \leq f\left(v_{m}\right)$. Then clearly $\sigma$ is a coding sequence of $G$ and $S=S_{\sigma}$. 
Theorem 9. Let $G$ be a graph with $s \geqslant 0$ isolated vertices, and let $k$ be the minimum number of prime factors of $\lambda(\sigma)$ among all coding sequences $\sigma$ of $G$. Then $\theta_{t}(G)=k+s$.

Proof. Let $\sigma$ be a coding sequence of $G$ such that the number of prime factors of $\lambda(\sigma)$ is $k$. Then by Lemma $6, k+s=\left|S_{\sigma}\right| \geqslant$ $\theta_{t}(G)$. Again let $S$ be a total clique covering of $G$ such that $|S|=\theta_{t}(G)$. Then by Lemma 8 , there is a coding sequence $\sigma$ of $G$ such that $S=S_{\sigma}$ and it follows from the proof of Lemma 8 that there are $|S|-s$ prime factors of $\lambda(\sigma)$. Thus, $k \leqslant|S|-s=$ $\theta_{t}(G)-s$. Therefore, $\theta_{t}(G)=k+s$.

Definition 10. Let $G=(V, E)$ be a graph with $|V|=m$ and $s \geqslant 0$ isolated vertices. Let $k=\theta_{t}(G)-s$. Let $S=$ $\left\{C_{1}, C_{2}, \ldots, C_{k+s}\right\}$ be a total clique covering of $G$ such that $|S|=\theta_{t}(G)$ and $\left|C_{i}\right|=1$ for $i=1,2, \ldots, s$. Now as in the proof of Lemma 8 , there are $k$ ! ways of assigning the first $k$ primes to the cliques $C_{s+1}, C_{s+2}, \ldots, C_{s+k}$ to obtain at most $k$ ! different coding sequences. Let $\sigma[S]$ be the least among them in the lexicographic ordering in $\mathbb{R}^{m}$. Then $\sigma[S]$ is called the coding sequence of $G$ with respect to $S$. Let $\left\{S_{1}, S_{2}, \ldots, S_{r}\right\}$ be the set of all total clique coverings of $G$ such that $\left|S_{i}\right|=\theta_{t}(G)$ for all $i=1,2, \ldots, r$. Let $\sigma(G)$ be the least element of $\left\{\sigma\left[S_{i}\right] \mid i=\right.$ $1,2, \ldots, r\}$ in the lexicographic ordering in $\mathbb{R}^{m}$. Then $\sigma(G)$ is called the code of the graph $G$.

For example, $(2,3,3,5,5,6,6,6,7,11,385)$ and $(2,3,3,5$, $5,7,10,10,10,11,231)$ are two coding sequences of the graph $G$ in Example 2 with respect to the total clique covering $S=$ $\left\{C_{1}, C_{2}, \ldots, C_{5}\right\}$. It is easy to see that $\theta_{t}(G)=5$ and $S$ is the only total clique covering with 5 cliques. The code of $G$ is $(2,2,3,3,5,7,10,10,10,11,231)$. Note that for any graph $G$, we have $G \cong G[\sigma(G)]$.

Theorem 11. Let $G_{1}$ and $G_{2}$ be two graphs. Then $G_{1} \cong G_{2}$ if and only if $\sigma\left(G_{1}\right)=\sigma\left(G_{2}\right)$.

Proof. The proof follows from Definition 10.

Definition 12. Let $G=(V, E)$ be a graph with $s \geqslant 0$ isolated vertices. Let $S=\left\{C_{1}, C_{2}, \ldots, C_{s+k}\right\}$ be a total clique covering of $G$, where $\left|C_{i}\right|=1$ for $i=1,2, \ldots, s$. If $v \notin C_{1} \cup C_{2} \cup \cdots \cup C_{s}$, let $m(v)=\prod\left\{x_{j} \mid v \in C_{s+j}\right\}$ be a monomial in the polynomial semiring $\mathbb{Z}_{0}^{+}\left[x_{1}, x_{2}, \ldots, x_{k}\right]$ of $k$ indeterminates over the semiring of nonnegative integers with usual addition and multiplication. For any $v \in C_{i}, i=1,2, \ldots, s$, define $m(v)=1$. Now we define

$$
f(G, S)=f\left(x_{1}, x_{2}, \ldots, x_{k}\right)=\sum_{v \in V} m(v) .
$$

Then $f(G, S)$ is said to be a polynomial representation of $G$ with respect to $S$.

Consider the graph $G$ in Example 2. Then $f(G, S)=x_{1}+$ $2 x_{2}+2 x_{3}+x_{4}+x_{5}+3 x_{1} x_{2}+x_{3} x_{4} x_{5}$ is a polynomial representation of $G$ with respect to $S=\left\{C_{1}, C_{2}, C_{3}, C_{4}, C_{5}\right\}$. Now let $G=(V, E)$ be a graph, and let $\sigma=\left(\lambda_{1}, \lambda_{2}, \ldots, \lambda_{m}\right)$ be a coding sequence of $G$. From the construction of $S_{\sigma}$ (cf. Lemma 6, Definition 7) and by Definition 12 , it follows that $f\left(G, S_{\sigma}\right)$ can also be obtained from $\sigma$ by replacing primes $p_{i}$ by $x_{i}(i<j \Leftrightarrow$ $\left.p_{i}<p_{j}\right)$ and commas by the addition symbol. It is important to note that the constant term of $f(G, S)$ in Definition 12 is the number of isolated vertices of $G$.

Definition 13. Let $G$ be a graph and $\sigma(G)$ the code of $G$. Then the polynomial representation of $G$ corresponding to $\sigma(G)$, that is, $f\left(G, S_{\sigma(G)}\right)$, is called the normal polynomial representation or the canonical polynomial representation of $G$ and is denoted by $F(G)$.

The normal polynomial representation of the graph $G$ in Example 2 is given by

$$
F(G)=2 x_{1}+2 x_{2}+x_{3}+x_{4}+x_{5}+3 x_{1} x_{3}+x_{2} x_{4} x_{5} .
$$

The following interesting observations are immediate from Definition 13.

Observation 1. A graph $G$ is disconnected if and only if $F(G)=f_{1}+f_{2}$, where $f_{1}$ and $f_{2}$ are polynomials with no common variables between them. The same is true for $f(G, S)$ for any total clique covering $S$ of $G$.

Observation 2. A graph $G$ is bipartite if and only if $F(G)=$ $f_{1}+f_{2}$, where monomials belonging to the same $f_{i}$ have no common variables, for $i=1,2$. The same is true for $f(G, S)$ for any total clique covering $S$ of $G$.

We now proceed to obtain a formula for $F(G(n))$.

Theorem 14. Let $n=p_{1}^{r_{1}} p_{2}^{r_{2}} \cdots p_{k}^{r_{k}} \in M$, where the $p_{i}$ 's are distinct primes. Then $\theta_{t}(G(n))=k$ and there is only one total clique covering of $G(n)$ with precisely $k$ cliques.

Proof. If $n$ is prime, then the result is obvious. Suppose $n$ is not a prime number. Now the vertices of $G(n)$ correspond to all the divisors of $n$, greater than 1 . So there are $k$ vertices labeled $p_{i}, i=1,2, \ldots, k$. Clearly, no two of them can lie in the same clique as $\operatorname{gcd}\left(p_{i}, p_{j}\right)=1$ for all $i \neq j$. So in any total clique covering of $G(n)$, these $k$ vertices will be in $k$ different cliques. In other words, any total clique covering has at least $k$ cliques. Now let $C_{i}=\left\{v \in V(G(n)) \mid p_{i}\right.$ divides $\left.v\right\}$. Then $S=\left\{C_{i} \mid i=\right.$ $1,2, \ldots, k\}$ is easily seen to be a total clique covering of $G(n)$. So we have a total clique covering consisting of precisely $k$ cliques. Hence, $\theta_{t}(G(n))=k$.

Now we show that there is only one total clique covering of $G(n)$ containing precisely $k$ cliques. Suppose there is another total clique covering $D=\left\{D_{1}, D_{2}, \ldots, D_{k}\right\}$ of $G$ with exactly $k$ cliques. Here also, the vertices labeled $p_{i}, i=$ $1,2, \ldots, k$, are in distinct cliques. Without loss of generality, let $p_{i} \in D_{i}$ for $i=1,2, \ldots, k$. Now for any $i, p_{i}$ is not adjacent to those vertices which are not in $C_{i}$. So $D_{i} \subseteq C_{i}$ for all $i=$ $1,2, \ldots, k$. Now suppose that for some $j$, there is a vertex $v$ in $C_{j}$ which is not in $D_{j}$. Now $D$ being a total clique covering, $v$ has to lie in at least one $D_{l}$, where $l \neq j$. Since a total clique covering covers all the edges, the edge between $v$ and $p_{j}$ will be covered. $v \notin D_{j}$ implies that $v$ and $p_{j}$ both lie in some $D_{l}$, where $l \neq j$. However, this contradicts the fact that $p_{j}$ is not adjacent to $p_{l}$. So $D_{i}=C_{i}$ for all $i=1,2, \ldots, k$ and hence $D=S$. 
Let $\alpha(G)$ denote the maximum size of an independent set in a graph $G$. In general, $\theta_{t}(G) \geqslant \alpha(G)$ for any graph $G$. The proof of the following proposition is similar to that of Theorem 14 and so it is omitted.

Proposition 15. Let $G$ be a graph with $\alpha(G)=k$. If $\left\{v_{1}, v_{2}, \ldots, v_{k}\right\}$ is an independent set and $S=\left\{S_{1}, S_{2}, \ldots, S_{k}\right\}$ is a total clique covering of $G$ such that each $v_{i}$ lies only in $S_{i}$ among cliques in $S$ for each $i=1,2, \ldots, k$, then $\theta_{t}(G)=\alpha(G)=$ $k$, and $S$ is the only total clique covering containing exactly $k$ cliques.

Now we provide a formula for $F(G(n))$.

Theorem 16. Let $n=p_{1}^{r_{1}} p_{2}^{r_{2}} \cdots p_{k}^{r_{k}} \in M$, where the $p_{i}$ 's are distinct primes and $r_{1} \geqslant r_{2} \geqslant \cdots \geqslant r_{k} \geqslant 1, k \geqslant 1$. Then $F(G(n))$ contains all the monomials $x_{i_{1}} x_{i_{2}} \cdots x_{i_{s}}$, where $\left\{i_{1}, i_{2}, \ldots, i_{s}\right\} \subseteq$ $\{1,2, \ldots, k\}, s \geqslant 1$ with the coefficient $r_{i_{1}} r_{i_{2}} \cdots r_{i_{s}}$, that is,

$$
F(G(n))=\sum_{\substack{i_{1}<i_{2}<\cdots<i_{s} \\ \emptyset \neq\left\{i_{1}, i_{2}, \ldots, i_{s}\right\} \subseteq\{1,2, \ldots, k\}}} r_{i_{1}} r_{i_{2}} \cdots r_{i_{s}} x_{i_{1}} x_{i_{2}} \cdots x_{i_{s}},
$$

unless $n$ is prime. If $n$ is prime, then $F(G(n))=1$.

Proof. If $n$ is prime, then $G(n) \cong K_{1}$ and so $F(G(n))=1$. Suppose $n$ is not prime. Now by Theorem $14, \theta_{t}(G(n))=k$ and there is only one total clique covering, say, $S$ of $G(n)$ with precisely $k$ cliques. Let $S=\left\{C_{1}, C_{2}, \ldots, C_{k}\right\}$, where $C_{i}=\{v \in$ $V(G(n)) \mid p_{i}$ divides $\left.v\right\}$ for $i=1,2, \ldots, k$. So $\sigma(G(n))$ is $\sigma[S]$. First, let $k>1$. Now $\sigma[S]$ is the least (in the lexicographic ordering) among the coding sequences of $G$ obtained from $S$. By Definition 10, any such coding sequence involves the primes $\left\{t_{1}, t_{2}, \ldots, t_{k}\right\}$, where $t_{i}$ is the $i$ th prime. Now for any $p_{i}$, there are $r_{i}$ elements (namely, $p_{i}, p_{i}^{2}, \ldots, p_{i}^{r_{i}}$ ) which belong to only the clique $C_{i}$. So considering the labellings and the fact that $r_{1} \geqslant r_{2} \geqslant \cdots \geqslant r_{k} \geqslant 1$, it is easy to see that the coding sequence will be the least in the lexicographic ordering if we assign $t_{i}$ to the clique $C_{i}$. So the vertex corresponding to a number $p_{i_{1}}^{q_{i_{1}}} p_{i_{2}}^{q_{i_{2}}} \cdots p_{i_{s}}^{q_{i_{s}}}\left(1 \leqslant q_{i_{j}} \leqslant r_{i_{j}}\right.$ for $j=1,2, \ldots, s$ where $1 \leqslant s \leqslant k)$ is assigned the label $t_{i_{1}} t_{i_{2}} \cdots t_{i_{s}}$. In other words, to find out $\sigma(G(n))$, we first consider the set of all divisors $\left\{m_{1}, m_{2}, \ldots, m_{r}\right\}$ (say) of $n$, greater than 1 , where $r=\left(r_{1}+\right.$ $1)\left(r_{2}+1\right) \cdots\left(r_{k}+1\right)-1$. Then in each $m_{j}$, we first replace $p_{i}$ by $t_{i}$, then make the resultant entries square-free and arrange them in nondecreasing order. This gives us $\sigma(G(n))=$ $\left(\lambda_{1}, \lambda_{2}, \ldots, \lambda_{r}\right)$, where each $\lambda_{i}$ is a product of primes of the form $t_{i_{1}} t_{i_{2}} \cdots t_{i_{s}},(1 \leqslant s \leqslant k)$ and this particular number repeats, say, $v$ times in the sequence $\sigma(G(n))$, where $v$ is the number of divisors of $n$ (greater than 1) which are of the form $p_{i_{1}}^{q_{i_{1}}} p_{i_{2}}^{q_{i_{2}}} \cdots p_{i_{s}}^{q_{i_{s}}}, 1 \leqslant q_{i_{j}} \leqslant r_{i_{j}}$ for $j=1,2, \ldots, s$; that is, $v=$ $\prod_{j=1}^{s} r_{i_{j}}$.

Thus, by Definition 13 we have $F(G(n))=$ $\sum_{i_{1}<i_{2}<\cdots<i_{s}, \emptyset \neq\left\{i_{1}, i_{2}, \ldots, i_{s}\right\} \subseteq\{1,2, \ldots, k\}} r_{i_{1}} r_{i_{2}} \cdots r_{i_{s}} x_{i_{1}} x_{i_{2}} \cdots x_{i_{s}}$.

Finally, if $k=1, r_{1}>1$, then $G(n) \cong K_{r_{1}}$. So $F(G(n))=$ $r_{1} x_{1}$, which satisfies the aforementioned formula. This completes the proof.
For example, $F\left(G\left(p^{2} q r\right)\right)=F(G(60))=2 x_{1}+x_{2}+x_{3}+$ $2 x_{1} x_{2}+2 x_{1} x_{3}+x_{2} x_{3}+2 x_{1} x_{2} x_{3}$, where $p, q$, and $r$ are distinct primes. Further, one may easily verify codes and normal polynomial representations for the following special classes of graphs:

(i) $\sigma\left(K_{1}\right)=(1), F\left(K_{1}\right)=1, \sigma\left(K_{n}\right)=(2,2, \ldots, 2)(n$ times), $F\left(K_{n}\right)=n x_{1}$ for $n \geqslant 2$,

(ii) $\sigma\left(P_{n}\right)=\left(p_{1}, p_{2}, p_{1} p_{3}, p_{2} p_{4}, \ldots, p_{n-3} p_{n-1}, p_{n-2} p_{n-1}\right)$, $F\left(P_{n}\right)=x_{1}+x_{2}+\sum_{i=1}^{n-3} x_{i} x_{i+2}+x_{n-2} x_{n-1}$ for $n \geqslant 3$,

(iii) $\sigma\left(C_{n}\right)=\left(p_{1} p_{2}, p_{1} p_{3}, p_{2} p_{4}, \ldots, p_{n-2} p_{n}, p_{n-1} p_{n}\right)$, $F\left(C_{n}\right)=x_{1} x_{2}+x_{1} x_{3}+\sum_{i=1}^{n-2} x_{i} x_{i+2}+x_{n-1} x_{n}$ for $n \geqslant 4$,

where $p_{i}$ is the $i$ th prime for $i=1,2, \ldots, n$ and $K_{n}, P_{n}$, and $C_{n}$ are, respectively, the complete graph, the path, and the cycle with $n$ vertices.

\section{Conclusion}

There are some representations of simple undirected graphs in terms of adjacency matrices, adjacency lists, and unordered pairs which are not unique for isomorphic graphs. There are some other instances for unique representations [2,3]. In the nauty algorithm, McKay [2] defined a canonical isomorph [4] which is a graph rather than a sequence of integers as the code of a graph introduced here. The importance of the code $\sigma(G)$ is its uniqueness and its simple form. It is the same for any set of isomorphic graphs. The determination of $\sigma(G)$ is not always easy, but once it is obtained for a graph, it becomes the characteristic of the graph. The authors believe that further study of the code and the normal polynomial representation of a simple undirected graph will be helpful in further research on graph theory. The purpose of this paper is to communicate these interesting observations to all graph theorists.

\section{Acknowledgment}

The authors gratefully acknowledge the learned referees for their kind suggestions and comments which enriched the paper.

\section{References}

[1] I. Chakrabarty, S. Ghosh, T. K. Mukherjee, and M. K. Sen, "Intersection graphs of ideals of rings," Discrete Mathematics, vol. 309, no. 17, pp. 5381-5392, 2009.

[2] B. D. McKay, "Practical graph isomorphism," Congressus Numerantium, vol. 30, pp. 45-65, 1981.

[3] R. P. Stanley, Enumerative Combinatorics, Cambridge University Press, Cambridge, UK, 2011.

[4] S. G. Hartke and A. J. Radcliffe, "McKay's canonical graph labeling algorithm," in Contemporary Mathematics, vol. 479, pp. 99111, American Mathematical Society, Providence, RI, USA, 2009. 


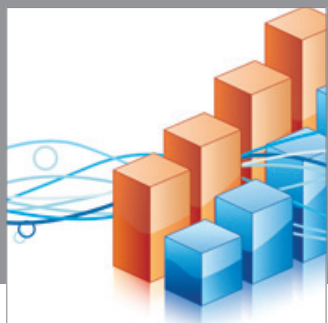

Advances in

Operations Research

mansans

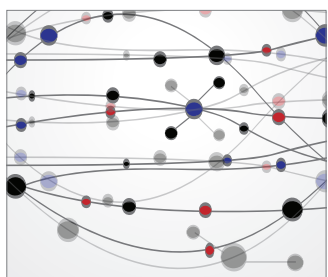

The Scientific World Journal
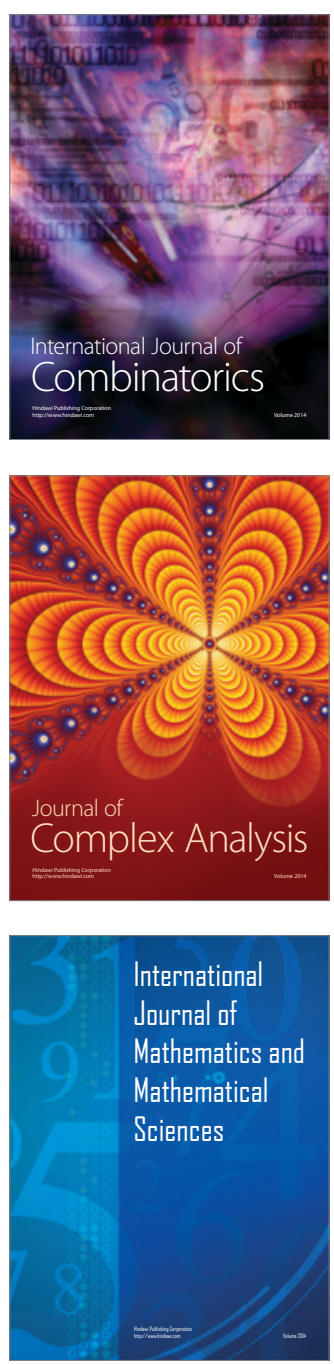
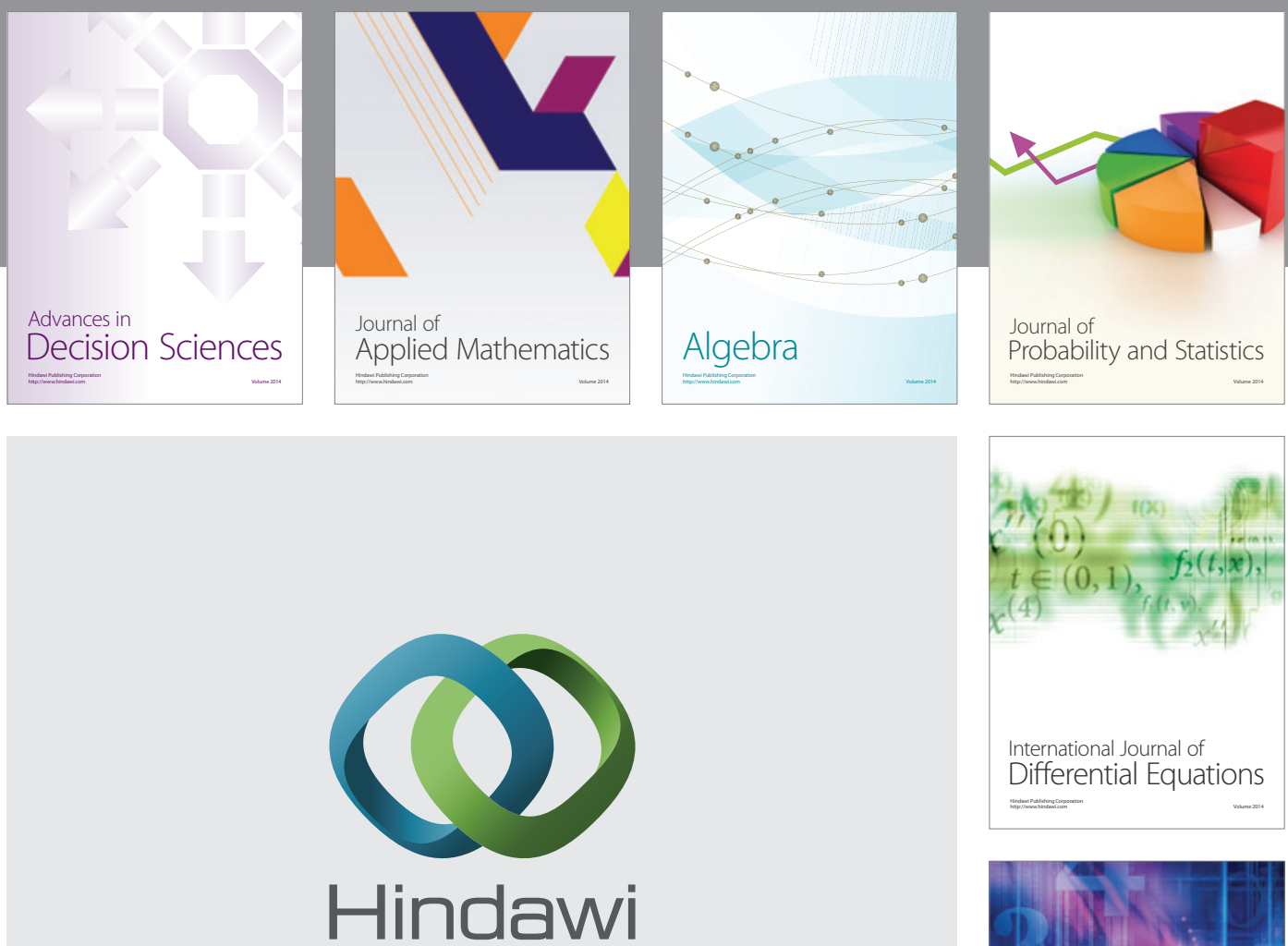

Submit your manuscripts at http://www.hindawi.com
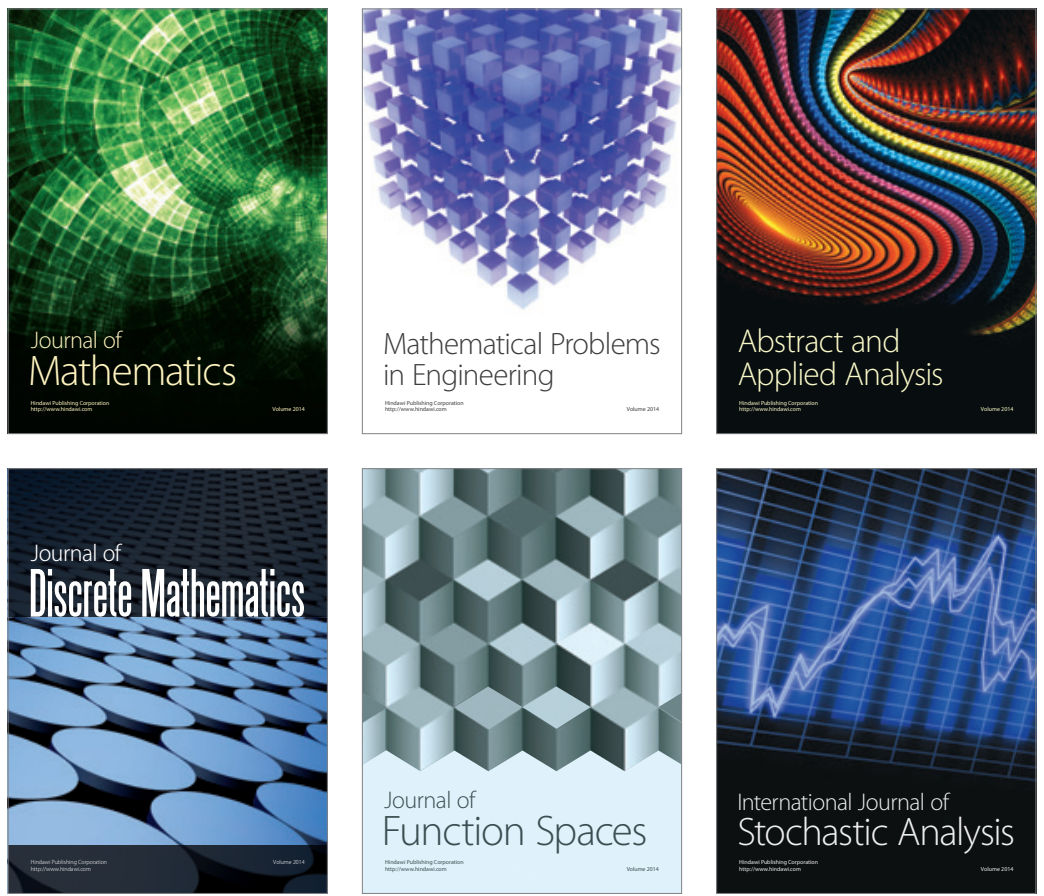

Journal of

Function Spaces

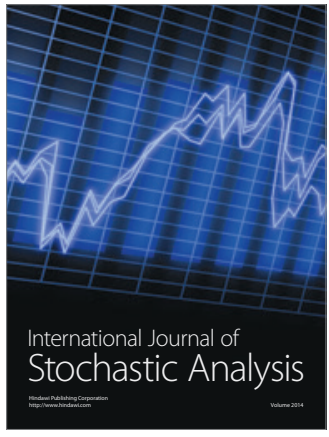

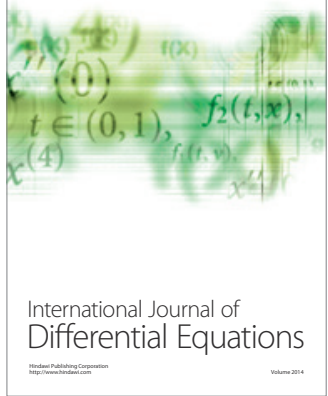
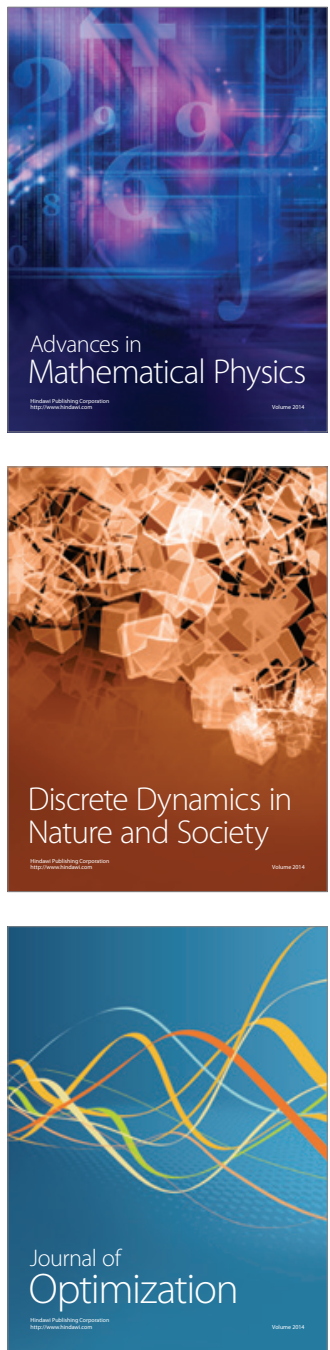